\title{
Preparing for Merger: An Art and Science Approach for Organisational Development
}

\author{
Harold Schroeder \\ Schroeder \& Schroeder, Inc. \\ 1 Yonge Street, Suite 1801, Toronto, Ontario M5E 1W7 \\ Tel: 1-416-244-0892Ｅ-mail: Harold@ schroeder-inc.com
}

\author{
Accepted: December 15, 2012 Published: January 31, 2013 \\ Doi:10.5296/ijhrs.v3i1.3185 URL: http://dx.doi.org/10.5296/ijhrs.v3i1.3185
}

\begin{abstract}
High failure rates continue to be reported for organizational change projects in general and mergers and acquisitions in particular, with the failures most often attributed to cultural incompatibilities or other people-related issues. The article identifies factors believed to be contributing to this situation, which mean that organisations are often inadequately prepared for the challenges of merger and integration. The Art and Science of Transformation ${ }^{\mathrm{TM}}$ framework developed by Schroeder \& Schroeder, Inc. and two related organizational change readiness assessment tools are discussed as a suggested approach for improvement of the prospects for a successful merger. These enable organizations considering a merger to determine their current state of change readiness in terms of art and science skills as well as proven organizational change shaping levers, and identify the cultural and people-related factors that can facilitate or hinder transformation. In this way, potential risks can be identified and eliminated and key drivers of successful transformation can be strengthened.
\end{abstract}

Keywords: Mergers, Organizational Transformation, Art and Science

\section{Introduction}

For many years now, organizational development specialists have been flagging up the importance of the people-related aspects of organizational transformation, and the risks of neglecting this dimension of change. With this increased awareness of the "art" of change, the question remains as to why it is still not effectively being applied. High failure rates continue to be reported for organizational change projects in general and mergers and acquisitions in particular, with the failures most often attributed to cultural incompatibilities or other people-related issues.

In this article, I identify a number of factors that I believe to be contributing to this situation, and which mean that organisations are often inadequately prepared for the challenges of merger and integration. As a suggested approach for improvement of the prospects for a successful merger, I discuss the Art and Science of Transformation ${ }^{\mathrm{TM}}$ framework developed by Schroeder \& Schroeder, Inc. as well as two related organizational change readiness assessment tools. These enable organizations considering a merger to determine their current state of change readiness in terms of art and science skills as well as proven organizational 
change shaping levers, and to identify the cultural and people-related factors that can facilitate or hinder transformation. In this way, potential risks can be identified and eliminated and key drivers of successful transformation can be strengthened.

Exploring the use of art in mergers and acquisitions further, I discuss the importance of art in identifying core project values, which can help overcome the differences in national and corporate culture which often hinder the success of a merger. Further, I consider the importance of measuring the return on investment (ROI) of the "art" of transformation, to provide a more robust evidence base for this approach.

\section{The Art and Science of Transformation}

This approach is based on the understanding, developed from an extensive review of the business research literature as well as decades of first-hand experience of assisting organizations with transformation projects, that successful change requires the application of both art and science.

The science of transformation involves expertise in the tools and techniques that form the basis of project management and business analysis training. For example, in a merger and acquisition situation, important aspects of science include the ability to develop a detailed change management plan, accurately estimate the required budget for the post-merger integration, identify and assess the potential risks to a successful merger and develop performance metrics and a system for monitoring project progress.

However, the ability to apply this science effectively in the real-life rather than the textbook context requires the ability to interact with and influence people. This is never more true than when undergoing a merger, since the nature of these changes have a big impact on the employees of the organizations involved, resulting in attitudinal and behavioral responses that can have a significant impact on the project outcomes. There is a need not only to manage these attitudes and behavior but also to reconcile organizational culture differences - which heavily influence ways of thinking and doing things in aspects of the business - and to secure the engagement and commitment of employees to a new shared vision and goals. All this requires the types of skills and expertise that are not easily taught in formal education and training, but derive from personal attributes or are developed over time from from experiential, on-the-job learning and observation. For example, the art of transformation includes the types of skills and abilities associated with strong leadership, the ability to build and motivate teams, communicate effectively in speech and writing with diverse audiences and draw on one's own initiative when faced with difficulties and challenges. It also includes the types of intangible, instinctive abilities that often prove to be so insightful and valuable at work, and can be defined in terms such as people acumen, business acumen and emotional intelligence. Whereas the science of transformation is often associated with left-brain thinking, or a logical, analytical approach, the art of transformation draws on right-brain thinking, which tends to be more holistic, creative, and intuitive.

Art and science are important for success in any organizational transformation, but the required balance of skills in any specific project will vary, depending on its complexity and the numbers of stakeholders that are involved in the project and can influence the outcomes. Logistically complex projects with low levels of stakeholder involvement, such as the introduction of an enterprise-wide information system, may require more science than art, 
whereas an HR project to introduce a new type of staff performance measurement system might be logistically straightforward but the need to achieve acceptance and understanding of the system by all employees may call for a significant level of art inputs. Many mergers and acquisitions are both logistically complex and directly impact on and involve large numbers of people - thus requiring high levels of art and science (Figure 1).

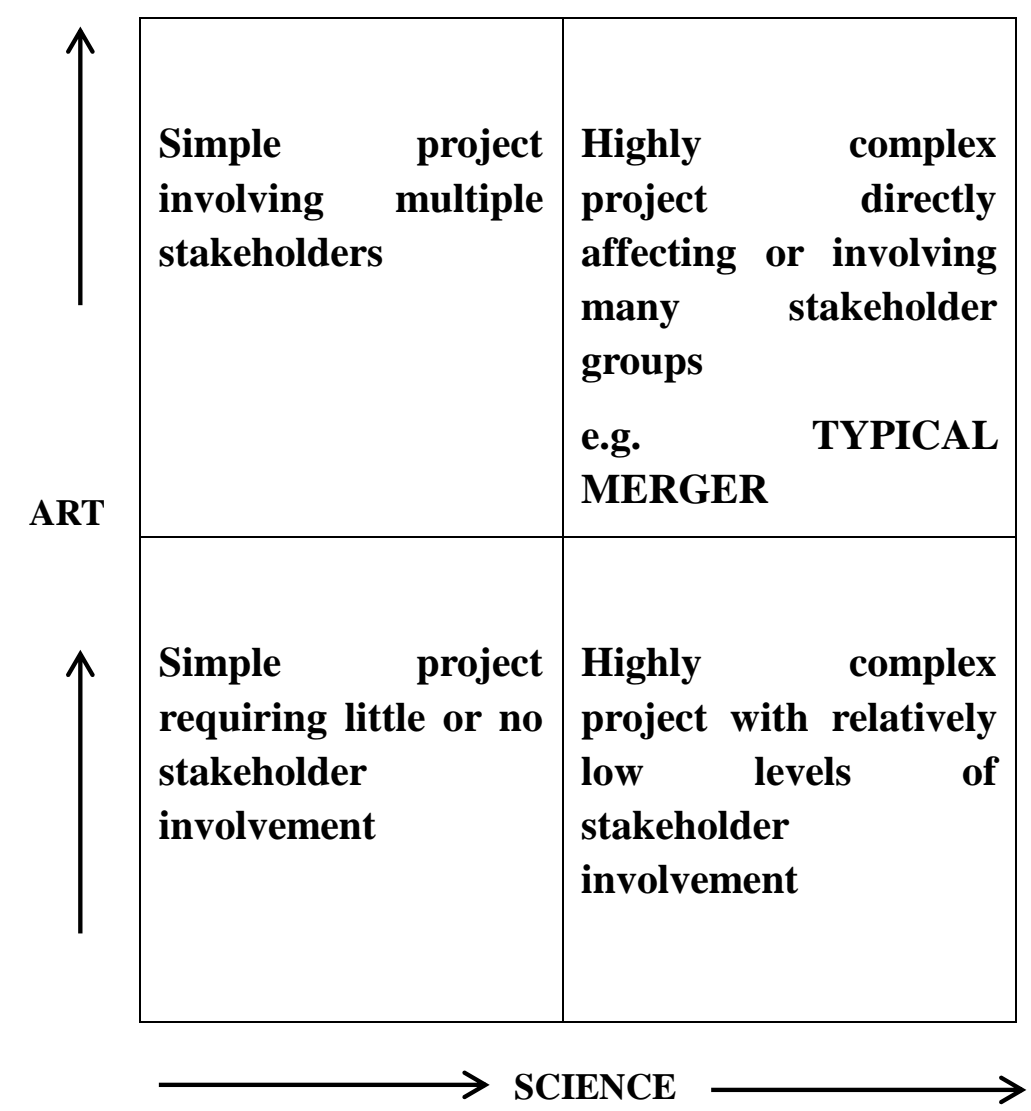

Figure 1: Relationship between Art and Science Skills and Types of Change Projects

\section{Why Mergers Fail}

The evidence is mounting that, when mergers fail, this most often results from a lack of attention to the art of change. There are numerous published case studies of failed or struggling mergers (e.g. Cheng \& Seeger, 2011; Fletcher, 2006; Lucas \& Rappeport, 2011), often involving high profile global corporations, which cite cultural conflicts as the main cause of the problems. This is also true of organizational change projects more generally; many studies have reported evidence that issues relating to organizational culture and employee resistance to change are common barriers to successful project implementation (e.g. Economist Intelligence Unit, 2009; IBM Corporation, 2008). Despite this growing body of research evidence, the continuing high failure rates being reported for mergers and acquisitions suggests that inadequate organizational learning is being gleaned from the mistakes of others to be applied in new mergers. I contend that there are two main reasons for this.

First, paying adequate attention to the people and cultural-related aspects of change is likely 
to have significant resource implications, especially if there is a need to recruit staff or employ external consultants, or provide training and development in the skills needed to effectively manage the people-related aspects of the merger. The difficulty in justifying these additional costs is that there is little concrete evidence of the likely return on investment (ROI). This is partly because the research "evidence" of the risks of neglecting people-related factors is mostly either anecdotal, based on individual case studies with which other organizations don't easily identify, or derived from relatively small and unscientific employer surveys. It can also be problematic to measure the more qualitative outputs arising from the art of transformation, and to reconcile these with traditional approaches to calculating ROI which rely on "hard" quantitative evidence such as financial profits or numerical measures of productivity. Improvements in employee engagement and motivation, increased brand awareness, company reputation and customer satisfaction are all qualitative dimensions of business which are more likely to be influenced by the art rather than the science of change, but which are not always easy to quantify in strictly financial terms. Failure to take account of their impact, however, can easily undermine the case for the necessary investment in the art of transformation, which is crucial to prepare organizations for a successful merger.

Second, prevailing approaches to business and project management are heavily science-based, relying on the application of formal tools and techniques. For example, the project management discipline has evolved from sectors such as construction in which project implementation requirements have typically been more predictable and amenable to the use of standardized approaches. More complex transformation projects, including most mergers, require not only good knowledge and expertise in the tools and techniques of change management, but also the ability to understand, interact with and influence a wide range of stakeholders in order to achieve the project goals. Unfortunately, this tends to be neglected, especially during the pre-merger negotiation stage when an increased focus on art would enable the prospective acquirer to identify any cultural-related risks or likely difficulties that could affect the ability to achieve the anticipated business benefits. In the due diligence process used to decide whether to proceed with a merger, financial and legal aspects are usually the main considerations and the question of cultural compatibility is often overlooked, sometimes with disastrous consequences once the merger goes ahead. Research has revealed that, ironically, though many employers see cultural compatibility as the greatest challenge in post-merger integration, few investigate this during the due diligence process (Horwitz et al, 2002).

\section{Assessing Organizational Change Readiness}

Many employers undergoing a merger realize with hindsight that they had not planned adequately for the integration stage, and seriously under-estimated the budgetary requirements and timescale for integration. Often, organizations are not adequately prepared for the major challenge of integration and lack the necessary skills, functions and processes needed to achieve this. There are two main aspects of change readiness that are crucial to investigate in the pre-merger phase, in order to ensure that all critical success factors for a successful post-merger integration are in place when the deal goes ahead. These are the change readiness of the project managers and other key staff who will be responsible for implementing the post-merger integration, and the change readiness of the merging 
organizations in terms of functions, processes and culture. In the following sections I describe as illustrative examples two change readiness tools developed by Schroeder and Schroeder, Inc. for use in investigating these two areas.

\subsection{The Project Manager Assessment System (PMAS)}

Project managers play a pivotal role in post-merger integration. According to the mergers and acquisitions literature, best practice project management in this area requires the appointment of a senior-level integration manager, as well as project teams responsible for the integration of different functional areas of the organization or aspects of the merger, and each headed by an experienced project manager (Bohlin, Daley \& Thomson, 2000; Daniel, 1999). The integration manager and their team will be responsible for ensuring successful co-ordination of the work of all the separate integration teams to ensure a successful merger, while other project managers will be responsible for developing and implementing integration plans for their own areas, and for resolving any difficulties or problems in order to achieve this. As highlighted by my earlier discussion of the Art and Science of Transformation, this means that project managers will need to possess high levels of both art and science skills.

The PMAS involves a unique approach to assessing the project management capability of an organization in terms of art and science, based on the understanding that successful project management requires not only the technical or "science" skills of project management but the softer "art" skills relating to human behaviour and interactions. While "science" skills may be evaluated by testing or on the basis of qualifications held, "art" or "soft" skills are generally more difficult to assess, and require qualitative data collection methods such as interviews or self-completion questionnaires requiring textual or essay type responses. This approach can also be helpful in exploring the actual ability of a project manager to use science as well as art skills in real project situations, by generating actual evidence of this in their current or previous job roles. The PMAS therefore incorporates a range of quantitative and qualitative methods, including an online questionnaire that collects both factual information on previous work experience and a self-assessment against specified science and art skills using a Likert scale; a psychometric test, and in-depth interviews conducted by external consultants.

Additional methods such as 360 degree assessment are also sometimes used, and are particularly helpful in generating information on the art dimensions of project management such as interpersonal interaction and leadership skills. In this way, project managers can be assessed not only on their knowledge and skills but on the ability to apply these effectively to various types of project management situation. Individual project manager assessment results can then be aggregated at team, department or organizational level, allowing the ability to identify strengths and weaknesses in skills and expertise in different areas or within the organization as a whole, and take steps to address these by the allocation of people to posts when they are most needed, or by additional recruitment or training as necessary. By tailoring the specific content of the assessment to organizational needs, such as the particular issues and challenges expected to arise in post-merger integration it is possible to ensure that the right skills and expertise are available to ensure the achievement of a successful outcome.

\subsection{The Organizational Change Management Assessment System (OCRAS)}

Complementary to the PMAS, the Organisational Change Readiness Assessment System (OCRAS) enables an organization to examine its systems, processes and organizational 


\section{Macrothink Institute ${ }^{T M}$}

culture and - as soon as possible - those of the acquisition organization, to identify any potential problems or weaknesses that may undermine post-merger integration efforts and to ensure that the appropriate pre-conditions for 1 transformation are in place. Again, a multi-methods approach is used to provide comprehensive and insightful information on strengths, weaknesses and the types of improvements that need to be made to help ensure a successful transition. In the case of OCRAS, this involves a review of organizational structure and relevant documentation, as well as in-depth interviews with a range of key informants in relevant areas of the organization. There are two main components to the assessment.

The first component focuses on understanding organizational change readiness in terms of a number of "change shaping levers" including communications methods and strategies, the overall structure of the organization, the compensation and rewards system, and the approach to performance management (Figure 2). These and other organizational factors have been identified from our experience of helping organizations under-go transformation, and from the business literature, as being especially important in driving and influencing change. Special attention therefore needs to be paid to these areas in a merger or other major change project, to ensure that they are designed to support the objectives and desired end result of the project, or to identify changes that are necessary to achieve these. In a merger, it is especially important to compare these areas across the two organizations involved, in order to identify differences that will need to be reconciled in order to achieve a successful integration.

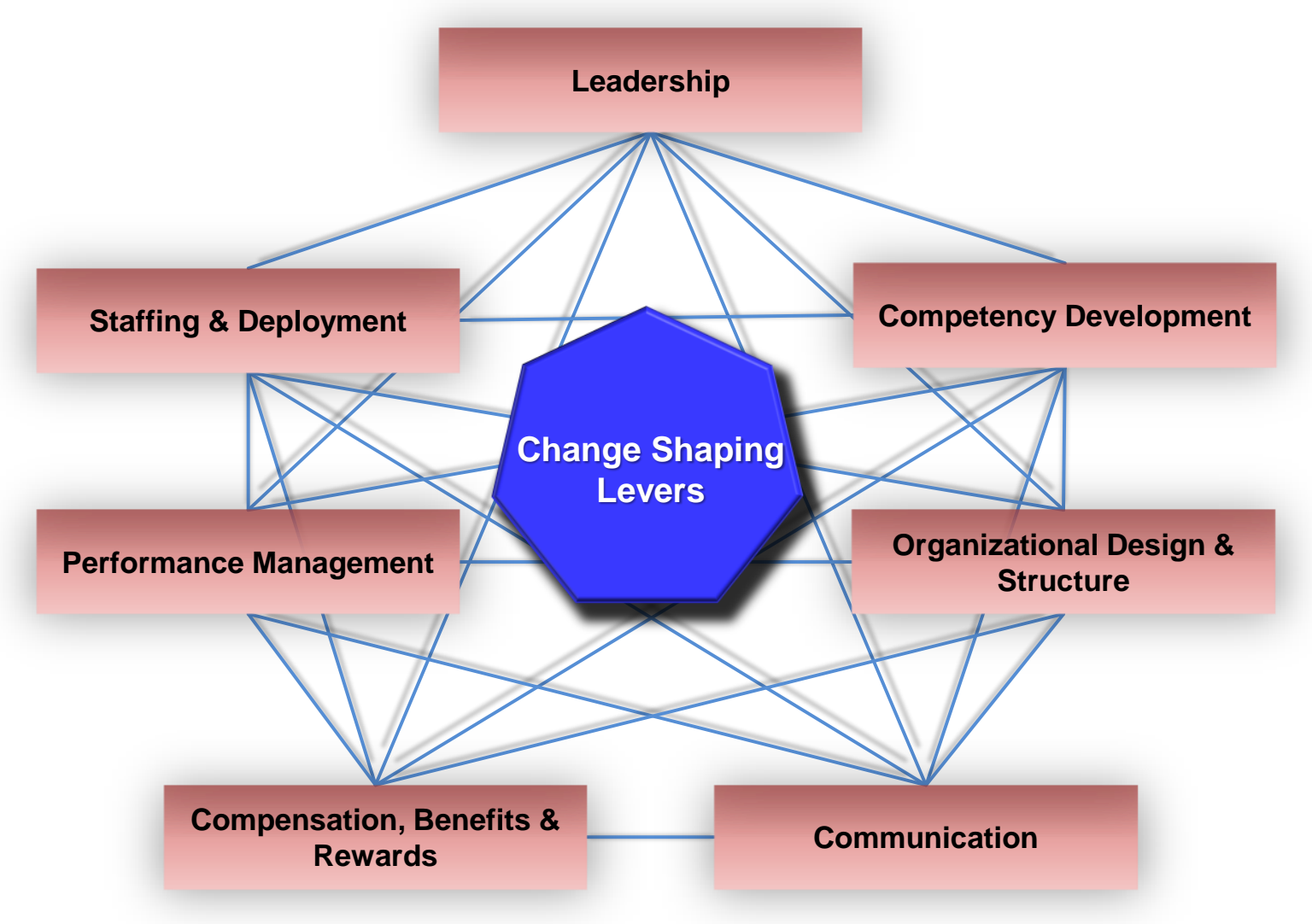

Figure 2. Change Shaping Levers in Organizational Transformation 
The second main component of the OCRAS uses an organizational climate assessment survey, as well as interviews with a sample of individuals from across the organization, at all levels of seniority, and a review of relevant documentation, to explore aspects of corporate culture, as well as other people-related factors that can either promote or hinder successful organizational transformation. This is especially important in the context of mergers, due to the commonly cited role of cultural incompatibilities in preventing successful integration. The types of issues explored in this stage of the assessment include, for example, leadership styles, levels of employee stress and anxiety, morale and motivation, and expectations and perceived risks relating to the impending change.

One of the main benefits of the multi-methods approach used in this system is the ability to generate quantitative data which enables organizations to prioritize and target their efforts and resources on the issues most likely to facilitate or hinder a successful merger, but also qualitative data which provides greater insights into how best to go about this.

In the case of the potential acquisition firm, it is of course often difficult or risky while negotiations are underway to gain access to employees for the purpose of cultural assessment research. However, it is nevertheless possible to elicit or infer a great deal of information about the culture of the organization from the executives involved in the merger discussions, from the narrative sections of annual reports, business plans or other published company literature. If the company is large and well known, a systematic review of the business literature may also reveal published articles, research or other information about the firm which shed light on aspects of corporate culture. The OCRAS therefore adopts a flexible, creative approach to securing relevant data on organizational culture, especially pertaining to the target firm in a prospective merger.

\section{The Role of Values in Change Readiness}

As highlighted earlier, cultural differences as well as other people-related factors such as resistance to change, often form the main barriers to successful post-merger integration, but are often unanticipated in the pre-merger stage (Boden, Guth, Heinze \& Lang, 2011; Vancea, 2011). Culture has been described in terms of values that determine the way that people think, feel and react, and which distinguish one group from another (Hofstede, 1983). Organizational cultures generally reflect national cultures but are also determined by other factors such as leadership and management styles which often vary considerably between companies even in the same country. They influence, for example, the governance and management structures and styles which exist in the organization, and how its members communicate with one another, deal with conflict, are involved in decision-making and are assessed and rewarded for their performance (Fletcher, 2006).

For example, in a merger project that Schroeder \& Schroeder consultants worked on in the Canadian Financial Services sector, two outwardly similar firms were found to have highly contrasting internal cultures - one based on a hierarchical structure and authoritative management style, the other on a flatter, team-based structure and high levels of employee empowerment. The differences were manifested in very different decision-making processes, levels of communication between leaders and staff, staff performance systems, etc. This gave rise to all sorts of difficult challenges in the post-integration merger, relating for example to the style and content of communications with staff about the merger, how best to combine 
very different performance assessment systems, and achieving similar levels of authority between people at the same grade. And this was within the same country and sector: the incompatibilities can be even more difficult to overcome in the case of cross-border mergers, when often a need to reconcile both corporate and national culture differences.

In our experience, one of the best ways to deal with this is to first establish common "culture-neutral" values, agreed on by senior executives of the respective merging organizations, which to guide the project and help facilitate stakeholder interactions and the establishment of common goals. Despite cultural differences between countries and organizations, there are certain relatively universal values which can be used to help promote a smooth transition and ensure that even when differences or conflicts arise they can be dealt with in ways which minimize risk to the project.

However, this brings us back to the importance of art skills in effective post-merger integration. Understanding the respective organizational cultures and identifying suitable common goals requires astute art skills and abilities, as does the ability to communicate these to project stakeholders and articulate them in ways that appeal to and engage people's commitment. The communication of values may be in the form of the project charter circulated to all integration team members or all employees, which highlights the role of these in underpinning the way the merger will be conducted. In the selection of project values there will be a need to ensure that these are not seen as reflecting the dominant culture of the acquiring organization, and do not conflict with any important values in the respective national cultures. For example, core project values reflecting individualism, such as assertiveness or a focus on individual goal achievement goals will probably be inappropriate in cultures where collectivism and team working are dominant values. More culture-neutral values may include, for example, honesty and trust, fairness, empathy, respect for others or reliability. Transparency and trust, in particular, have been highlighted in many studies as being crucial in securing the commitment of employees to a merger project, and require for example that organizational leaders openly communicate the reasons for their decisions and do not make commitments to staff that they cannot keep. This is a complex issue in many cross-border mergers, since acceptable levels of trust and transparency vary considerably between national cultures, but in general a focus on these types of values has been shown to be beneficial in most merger situations (Michalak, 2010; Vector Group, 1996).

\section{The ROI of Art in Transformation}

Just as it is now recognized that social and environmental factors in addition to financial factors contribute to the costs of doing business and value generated (the triple bottom line), there is equally a need for greater acknowledgement - in mergers and other organizational transformations - of the costs and risks associated with prevailing science-dominated approaches, and the likely ROI of an approach that incorporates art. As discussed in this paper, an art-focused approach is necessary to help resolve or reconcile cultural conflicts, establish a culture-neutral value system to underpin the processes of merger and integration, secure and maintain the engagement and commitment of employees and other stakeholders to a new shared vision and goals and communicate information in ways that help resolve concerns as well as motivating and inspiring people.

Though it can be quite difficult to quantify this in the financial terms traditionally associated 
with ROI (the science approach), there are alternative ways that the outcomes of a more art-focused approach can be monitored and their impact assessed, using both quantitative and qualitative measures. This is extremely important not only to demonstrate progress in the post-merger integration and to highlight any problem areas that need to be addressed, but also to support the case for any additional resources needed to improve art skills within the organization that emerge during the change readiness assessment.

According to a recent employer survey, the majority of companies undergoing a merger do systematically measure their integration performance using Key Performance Indicators. However, the emphasis tends to be mainly on financial measures of performance, and there is little focus on more qualitative indicators (PricewaterhouseCoopers, 2009). Other research has shown that those companies that achieve the smoothest integrations anticipate their desired end states at various future milestones, and develop both quantitative and qualitative measures of performance to assess progress towards these (Bohlin, Daley \& Thomson, 2000; Bourke, Laidlaw \& Woods, 2000/1). For example, a case study of an acquisition by BUPA Australia has discussed the use of non-financial measures of performance in a balanced scorecard system, including measures of customer satisfaction and "human capital scores" pertaining to employee engagement (Cascio, 2010). Other innovative measures of integration success, such as employee identification with the products and services of the newly merged organization (Bohlin, Daley \& Thomson, 2000), can also be developed. These can be incorporated in the types of organizational change readiness assessment tools discussed in this article, to provide both quantitative and qualitative benchmark data against which integration progress can be monitored. By applying a more art-focused approach to the assessment of integration success, it should be possible to identify the ways in which improvements in these qualitative areas are likely to be associated with overall business performance and generate evidence of the ROI of the art of transformation.

\section{Conclusion}

According to the Bloomberg 2012 Outlook, global M\&A activity is continuing to grow, with almost 25,000 new deals being made in the year to end November 2011, representing more than $\$ 2.11$ trillion in volume, an increase of 9\% from 2010 volume levels. If past experience is anything to go by, at least half of these deals may fail altogether, or fall considerably short of achieving the expected business goals and value, and many of these failures will be due to cultural or other people-related factors that were not anticipated in the pre-merger negotiations.

This is a situation which can be readily avoided by conducting more extensive change readiness assessments, and by recognizing and addressing the role of art as well as science in organizational change. This article has described how this approach can be employed in practice with examples of specific assessment systems that focus on the factors likely to make a real difference in post-merger integration. This may be the difference between success and failure in many mergers and acquisitions. 


\section{References}

Bloomberg (2012). $2012 \quad M \& A$ Outlook. [Online] Available: http://www.bloomberg.com/article/2011-12-08/a6a6z1oVzygI.html

Boden, C., Guth, E., Heinze, N. \& Lang, S. (2011). On the scent of culture - What has culture to do with Mergers and Acquisitions? [Online] Available: http://www.successacross.com/fileadmin/user_upload/pdfsdernews/2011-10/08.10.2011_Fina 1_News-story_english.pdf

Bohlin, N., Daley, E., \& Thomson, S. (2000). Successful post-merger integration: realizing the synergies. Handbook of Business Strategy, 1, 1, 225-231.

Bourke, E., Laidlaw, G.\& Woods, I. (2000/1). Achieving post-merger integration. Towers Perrin.

[Online]

Available:

http://www.towersperrin.com/tp/getwebcachedoc?webc=TILL/USA/2000/200003/200205151 1 .pdf

Cascio, W. (2010). Done deal: now manage post-merger integration. HRMagazine, 55, 10, 43-46.

Cheng, S.S. \& Seeger, M.W. (2011). Cultural differences and communication issues in international mergers and acquisitions: a case study of BenQ debacle. International Journal of Business and Social Science, 3, 3, 116-127.

Daniel, T. (1999). Between trapezes: The human side of making mergers and acquisitions work. Compensation \& Benefits Management,15, 1, 19-37.

Economist Intelligence Unit (2009). The burning platform: How companies are managing change. [Online] Available: http://www.celerantconsulting.com/ /media/PDF/Surveys/SV-Burning-platform.pdf Fletcher, A. (2006). Avoiding post-merger blues. Insight: Perspective. BearingPoint.

Hofstede, G. (1983). Culture's consequences: international differences in work-related values. Administrative Science Quarterly, 28, 4, 625-629.

Horwitz, F.,Anderssen, K., Bezuidenhout,A., Cohen, S., Kirsten, F.,Mosoeunyane, K., et al. (2002). Due diligence neglected: managing human resources and organizational culture in mergers and acquisitions. South African Journal of Business Management, 33, 1, 1-10.

IBM Corporation (2008). The Enterprise of the Future: IBM Global CEO Study 2008. [Online] Available: www.ibm.com/enterpriseofthefuture.

Lucas \& Rappeport (2011, May 23). Mergers and acquisitions: a bitter taste. FT.com. [Online] Available:

http://www.ft.com/cms/s/0/03559624-8571-11e0-ae32-00144feabdc0.html\#axzz1oM5Le1lw Michalak, J.M. (2010). Cultural catalysts and barriers of organizational change management: a preliminary overview. Journal of Intercultural Management, 2, 2, 26-36.

PricewaterhouseCoopers (2009). Post-Merger Integration Survey 2009: European Results. Author. [Online] Available: http://download.pwc.com/ie/pubs/post_merger_integration_survey_2009.pdf Vancea, M. (2011). Challenges and stakes of the post-acquisition integration process. Annales Universitatis Apulensis Series Oeconomica, 13, 1, 167-180.

Vector Group LCC (1996). Merging cultures. Author. [Online] Available: 
http://www.vectorgroupinc.com/documents/MergingCultures_VectorGroup_WorkingPaper.pd $\underline{\mathrm{f}}$ 